\title{
Stage IB Uterine Corpus Endometrial Stromal Sarcoma AJCC v8
}

National Cancer Institute

\section{Source}

National Cancer Institute. Stage IB Uterine Corpus Endometrial Stromal Sarcoma A/CC v8. NCI Thesaurus. Code C139885.

Stage IB includes: T1 b, N0, M0. T1 b: Uterine corpus endometrial stroma sarcoma with tumor limited to the uterus, measuring more than $5 \mathrm{~cm}$ in greatest dimension. NO: No regional lymph node metastasis. M0: No distant metastasis. (AJCC 8th Ed.) 